\title{
Dis Colon Rectum, Vol. 47, No. 12, December 2004, pp. 2032-2038 (DOI: 10.1007/s10350-004-0718-5)
}

Due to an electronic error in production, nine paragraphs of the Patients and Methods and Results section were omitted from the print and pdf versions of the article "The Efficacy of a Nerve Stimulator (Cavermap ${ }^{\circledR}$ ) to Enhance Autonomic Nerve Identification and Confirm Nerve Preservation During Total Mesorectal Excision" by Giovanna M. da Silva, M.D., Oded Zmora, M.D., Lars Börjesson, M.D., Nelly Mizhari, M.D., Norma Daniel, R.N., Farah Khandwala, M.S., Jonathan Efron, M.D., Eric G. Weiss, M.D., Juan J. Nogueras, M.D., Anthony M. Vernava III, M.D., Steven D. Wexner, M.D., published in the December 2004 issue of Diseases of the Colon E Rectum (Vol. 47, No. 12, pp 2032-2038, DOI 10.1007/s10350-004-0718-5). The HTML version is correct. Below, the entire article is printed in its entirety with the omitted paragraphs in bold format. Springer regrets the error.

Dis Colon Rectum 2005; 48: 2354-2361

DOI: $10.1007 / \mathrm{s} 10350-005-0224-4$

(C) The American Society of Colon and Rectal Surgeons

Published online: 13 October 2005

PURPOSE: Sexual dysfunction after total mesorectal excision may be caused by injury to the autonomic nerves. During surgery, nerve identification is not always achieved, and, to date, there has been no method to objectively confirm nerve preservation. The aim of this study was to assess the efficacy of a nerve-stimulating device (CaverMap ${ }^{\circledR}$ ) to assist in the intraoperative identification of the autonomic nerves during total mesorectal excision, and objectively confirm nerve preservation after proctectomy is completed. PATIENTS AND METHODS: Sexually active consecutive male patients undergoing total mesorectal excision were prospectively enrolled in this study. During pelvic dissection, the surgeon attempted to localize the hypogastric and cavernous nerves. Cavermap ${ }^{\circledR}$ was used to confirm these findings and to facilitate the identification in cases of uncertainty. At the completion of proctectomy, the nerves were restimulated to ensure preservation. Factors that could affect the surgeons ability to localize the nerves and Cavermap ${ }^{\circledR}$ to confirm this were evaluated. RESULTS: Twentynine male patients with a median age of 58 years were enrolled in this study. An attempt to visualize the hypogastric nerves during dissection was made in 26 patients; the surgeon was able to identify the nerves in 19 ( 73 percent)

Poster presentation at the meeting of The American Society of Colon and Rectal Surgeons, June 3 to 8, 2002, Chicago, Illinois.

Poster presentation at the meeting of the Association of Coloproctology of Great Britain and Ireland, July 2 to 5, 2002, Manchester, United Kingdom

Podium presentation at the Tripartite Colorectal meeting, October 27 to 30, 2002, Melbourne, Australia.

Correspondence to: Steven D. Wexner, M.D., Department of Colorectal Surgery, Cleveland Clinic Florida, 2950 Cleveland Clinic Blvd., 33331, Weston, Florida, e-mail: mcderme@ccf.org patients. Cavermap ${ }^{\circledR}$ successfully identified the nerves in six of the seven remaining patients, and failed to identify them in only one case. An attempt to localize the cavernous nerves during dissection was made in 13 patients, of which localization was successful in 8 (61.5 percent) patients. Cavermap ${ }^{\circledR}$ improved the identification rate in four of the remaining five patients. After proctectomy, Cavermap ${ }^{\circledR}$ successfully confirmed the preservation of both hypogastric and cavernous nerves in 27 of 29 (93 percent) patients. A history of previous surgery statistically correlated with failure to identify the hypogastric nerves by the surgeon $(P=$ 0.005). There were no adverse events related to use of the device. CONCLUSION: Cavermap ${ }^{\circledR}$ may be a useful tool to facilitate identification of the pelvic autonomic nerves during total mesorectal excision and to objectively confirm nerve preservation. [Key words: Impotence; Total mesorectal excision; Nerve stimulator; Rectal carcinoma; Suprapubic nerves; Parasympathetic nerves; Retrograde ejaculation]

$\mathrm{T}$ he incidence of sexual dysfunction after rectal surgery depends on several factors, including the age and gender of the patient, the nature of the disease, and the extent of the procedure. ${ }^{1}$ Male sexual dysfunction after pelvic surgery can manifest as impotence or ejaculation disorder. Previous studies have shown that the incidence of male sexual dysfunction after rectal surgery ranges between 14 and 18 percent in benign disease, ${ }^{2}$ and increases up to 59 percent in malignant disease. ${ }^{3}$ It is well known that preservation of the pelvic autonomic nerves during rectal surgery is crucial for the preservation of postoperative sexual 
function; the sympathetic nerves are essential for ejaculation, and the parasympathetic, for erection. ${ }^{3}$

Exact and sharp dissection has been shown to be oncologically fundamental and an integral part of total mesorectal excision (TME) and is standard among colorectal surgeons. ${ }^{4}$ This technique has been shown to facilitate autonomic nerve identification and preservation, subsequently improving postoperative sexual function. ${ }^{5}$ However, despite this being an optimal technique, exact identification of the pelvic autonomic nerves can sometimes be difficult for a variety of reasons, such as advanced disease, narrow pelvis, bleeding, and previous pelvic dissection, placing all autonomic nerves at risk for damage during rectal dissection.

CaverMap ${ }^{\circledR}$ Surgical Aid (CM; Blue Torch Medical Technologies, Inc., Ashland, MA) is a device designed to assist surgeons in intraoperatively localizing the autonomic nerves, ultimately leading to improved preservation of the autonomic nerves and sexual function. During radical prostatectomy, the use of CM has been shown to decrease the postoperative incidence of erectile dysfunction. ${ }^{6}$ The aim of this study was to assess the efficacy of CM in assisting the surgeon to identify the pelvic autonomic nerves during TME dissection and to objectively confirm nerve function intraoperatively after proctectomy.

\section{PATIENTS AND METHODS}

Twenty-nine consecutive sexually active male patients undergoing elective TME between October 2000 and September 2001 were prospectively enrolled in this study, which was approved by the Institution of Review Board of Cleveland Clinic Florida. Five board-certified colorectal surgeons performed all operations. Patients with an American Society of Anesthesiology score higher than III and/or carrying cardiac pacing equipment or other electromechanical prostheses were excluded from the study. Chemoradiation therapy was administered for patients with Stage II or III rectal cancer and consisted of 45.0 Gy in 25 fractions administered over a five-week period (1.8 Gy/fraction). This was followed by a boost to the primary tumor bed for a total dose of 50.4 Gy with concomitant continuous infusion of 5-fluorouracil (5FU) at a dose of $250 \mathrm{mg} / \mathrm{m}^{2} /$ day.

\section{CaverMap $^{\circledR}$ Device}

The device combines a nerve stimulator with an erectile response detection system for intraoperative

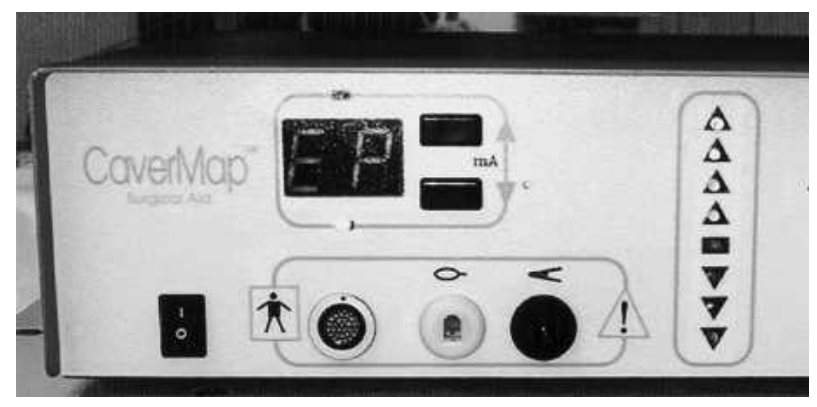

Figure 1. Control unit. The control unit contains the connectors for the probe handle and tumescence sensor. The unit displays the stimulation response with audible and visual signs.

use. The system is composed of a control unit, nervestimulating probe, and a tumescence sensor placed around the penis to measure changes in circumference (Figs. 1 and 2). During pelvic dissection, the probe was placed on the tissues to stimulate the nerves, eliciting a tumescence response of the penis, which was detected by the penile sensor that is able to detect minimal increases or decreases of 0.5 percent in penile diameter (Fig. 3). Changes in penile circumference were displayed in the control unit by audible and visible signals. The nerve stimulator electric output started at the default of $8 \mathrm{~mA}$, with stimulation set for 20 seconds. If no tumescence response occurred, the current was automatically gradually increased to a maximum of $20 \mathrm{~mA}$. If no response could be elicited at a maximum current level, the probe was repositioned. An increase or decrease in tumescence of 0.5 percent was considered a positive response.

\section{Pelvic Neuroanatomy}

The pelvic organs are innervated by sympathetic and parasympathetic fibers. The sympathetic supply arises from L1 to L3, which contribute to the preaortic superior hypogastric plexus that extends to the sacral promontory. At this level, the plexus gives origin to the right and left hypogastric nerves. The parasympathetic supply arises from S2 to S4. The fibers emerge through the sacral foramen (nerve erigentes) to join the sympathetic hypogastric nerves to form the inferior pelvic plexus at the pelvic sidewall at the level of the lower third of the rectum. From the pelvic plexus, nerve fibers (sympathetic and parasympathetic) extend to the different pelvic viscera. Some of these branches form the periprostatic plexus situated on Denonvilliers fascia, posterolateral to the prostate and seminal vesicles, where they are referred to as cavernous nerves and are responsible for erection. 


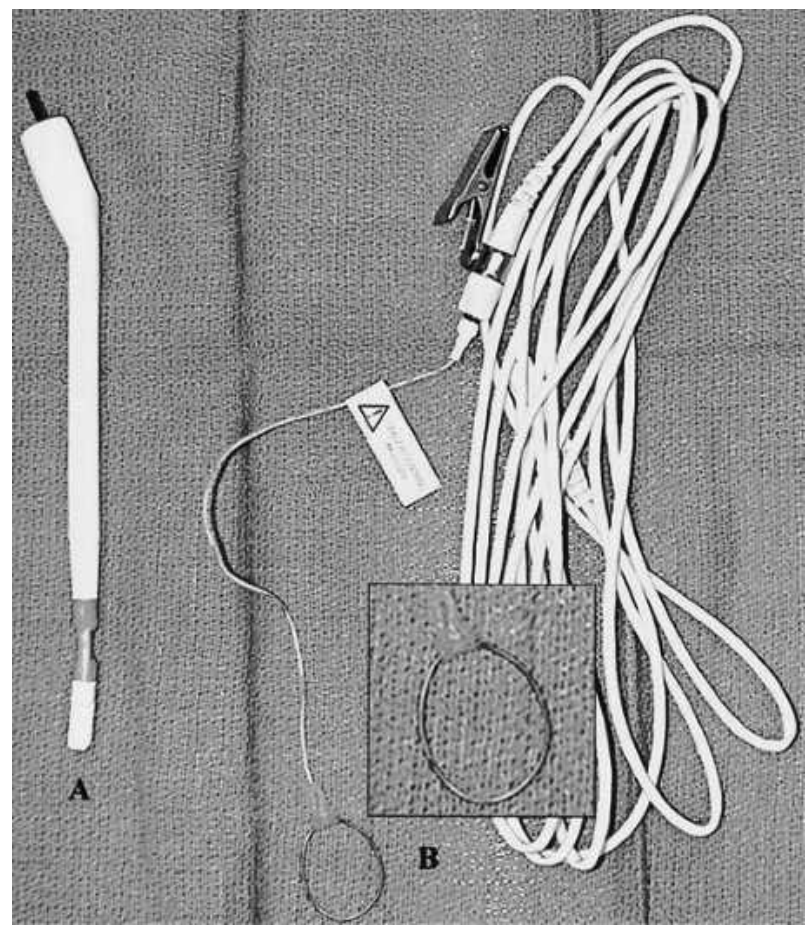

Figure 2. A. Probe handle. B. Tumesence sensor. During dissection, current is applied through the probe tip, resulting in penile tumescence that is detected by the sensor ring.

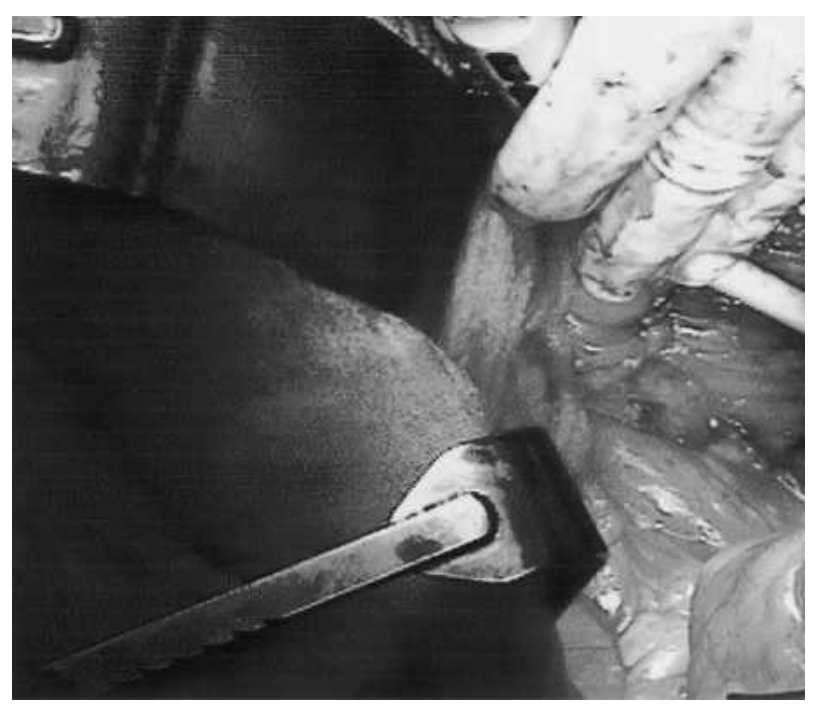

Figure 3. Intraoperative stimulation of the left hypogastric nerve.

In the current protocol, CM was used to stimulate the left and right sympathetic hypogastric nerves high on the pelvic sidewall (just after their origin from the hypogastric plexus on the sacral promontory) and the cavernous nerves located at the seminal vesicle level, where a positive CM response would exclude a proximal lesion of the nerves. The fact that stimulation of the sympathetic hypogastric nerves on the pelvic side wall was able to elicit a parasympathetic tumescence response may be explained by the flow of current down the nerve trunks to the pelvic plexus where synapses of the sympathetic and parasympathetic fibers occur.

\section{Surgical Technique, Identification, and Stimulation of Autonomic Nerves}

The TME surgical technique has been previously described in detail. ${ }^{7}$ Under general anesthesia and before the insertion of a Foley catheter, the tumescence sensor was fit around the base of the penis and connected to the control unit. During the pelvic dissection, the surgeon attempted to localize the right and left hypogastric nerves at the lateral sides of the pelvis. As the surgical procedure was continued inferiorly at the pelvis, the surgeon attempted to localize the cavernous nerves located at the posterolateral aspect of the seminal vesicles. These nerves were indirectly visualized, because they were covered by the Denonvilliers fascia. As the nerves were localized or suspected, they were stimulated and the penile tumescence response was reflected in the monitor screen and then recorded.

The ability of the surgeons to identify the autonomic nerves during dissection and the ability of $\mathrm{CM}$ to confirm these findings were assessed. The surgeons identification of the nerve before electric stimulation was reported as yes if they were certain about the nerve location, and no if they were unsure or were unable to localize the nerve at all. In cases of uncertainty, the ability of CM to facilitate the identification of the nerve was assessed. To assess possible nerve damage, the stimulation protocol was repeated after removal of the surgical specimen.

Possible impact of body mass index (BMI; $30 \mathrm{~kg} / \mathrm{m}^{2}$ or $<30 \mathrm{~kg} / \mathrm{m}^{2}$ ), preoperative radiation therapy, type of procedure (sphincter-saving surgery or proctectomy + end stoma), diagnosis (malignant, benign), and previous surgery affecting the surgeons ability to bilaterally identify the autonomic nerves and CM to confirm were assessed.

\section{STATISTICS}

Evaluation of selected factors that could possibly interfere with surgeons ability to locate the nerves was performed by use of exact unconditional tests for 
Table 1.

Stimulation During Dissection: Hypogastric Sympathetic Nerves

\begin{tabular}{|c|c|c|c|c|}
\hline \multirow[b]{2}{*}{ Surgeon Identification } & \multirow[b]{2}{*}{ Patients n (\%) } & \multicolumn{3}{|c|}{ CM Confirmation n (\%) } \\
\hline & & Bilateral & Unilateral & Negative \\
\hline Yes, bilateral & $17(65.3)$ & $16(94.1)$ & $1^{a}(5.9)$ & 0 \\
\hline Yes, unilateral & $2(7.7)$ & $2(100)$ & 0 & 0 \\
\hline No & $7(27)$ & $3(42.9)$ & $3(42.9)$ & $1(14.2)$ \\
\hline Total & $26(100)$ & $21(80.7)$ & $4(15.3)$ & $1(3.8)$ \\
\hline
\end{tabular}

$\mathrm{CM}=$ CaverMap $^{\circledR}$.

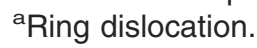

Table 2.

Stimulation During Dissection: Cavernous Nerves (Sympathetic/Parasympathetic)

\begin{tabular}{lcccc}
\hline & & \multicolumn{3}{c}{ CM Confirmation $\mathbf{n ~ ( \% ) ~}$} \\
\cline { 3 - 5 } Surgeon Identification & Patients $\mathbf{n ~ ( \% ) ~}$ & Bilateral & Unilateral & Negative \\
\hline Yes, bilateral & $7(53.8)$ & $4(57.1)$ & $2(28.6)$ & $1(14.3)$ \\
Yes, unilateral & $1(7.7)$ & $1(100)$ & 0 & 0 \\
No & $5(38.5)$ & $3(60)$ & $1(20)$ & $1(20)$ \\
Total & $13(100)$ & $8(61.5)$ & $3(23)$ & $2(15.3)$ \\
\hline
\end{tabular}

$\mathrm{CM}=$ CaverMap $^{\circledR}$.

contingency tables. A $\mathrm{P}$ value $<0.01$ was used for significance.

\section{RESULTS}

Twenty-nine patients with a median age of 58 (range, 40-67) years participated in the study. Fourteen patients were operated on for rectal cancer and 15 had various benign diseases (11 cases of ulcerative colitis, 2 of familial adenomatous polyposis, 1 of Crohns disease, and 1 of villous adenoma). Preoperatively, 24 patients were able to have intercourse, and 5 reported some degree of erection, although inadequate for intercourse; no patient was completely impotent.

During TME dissection (before rectal excision), 26 of 29 patients participated in the protocol surgeon nerve localization/CM confirmation regarding the hypogastric nerves, and 13 concerning the cavernous nerves. After rectal resection, all patients had the hypogastric nerves tested, 27 also had the cavernous nerves tested for any damage; no complications were related to use of the device.

\section{Hypogastric Nerves}

Table 1 summarizes the results of the sympathetic hypogastric nerve stimulation during dissection. The surgeon attempted to visualize the nerves in $26 \mathrm{pa}$ tients and was successful in 19 (73 percent; 17 bilat- eral and 2 unilateral). In the 17 patients in whom the nerves were bilaterally identified by the surgeon, CM was able to confirm function in all cases (bilaterally in 16 , and unilaterally in 1). In the patient with the initial unilateral response, the sensor ring had dislocated during dissection and an adequate response was elicited after repositioning of the ring. Furthermore, CM succeeded in identifying the contralateral nerve in all $(n=2)$ patients in whom it was only unilaterally located by the surgeon.

In seven (27 percent) patients, the surgeon was uncertain or could not identify the nerves at all; CM identified the nerves in six ( 85 percent; 3 bilateral and 3 unilateral) and completely failed to identify them in only one patient. Finally, in one patient, CM was used to exclude a neural structure and thereby facilitated the dissection. The mean number of attempts to elicit an adequate response from the hypogastric nerves was 1.3 (range, $1-7$ ).

\section{Cavernous Nerves}

Table 2 summarizes the results of cavernous nerve stimulation. Attempts to visualize these nerves during dissection were made in only 13 patients according to the surgeons preference. The nerves were identified in eight (62 percent) patients ( 7 bilaterally and 1 unilaterally). Among the seven patients in whom the nerves were bilaterally identified, CM confirmed both nerves in four patients and one of the nerves in two 
Table 3.

Stimulation of Autonomic Nerves After Proctectomy

\begin{tabular}{ccccc}
\hline & & & \multicolumn{2}{c}{ CM Confirmation $\mathbf{n}(\%)$} \\
\cline { 3 - 5 } Nerve & Patients $\mathbf{n}(\%)$ & Bilateral & Unilateral & Negative \\
\hline Hypogastric & $29(100)$ & $27(93)$ & $1(3.5)$ & $1(3.5)$ \\
Cavernous nerves & $27(93.1)$ & $23(85)$ & $3(11)$ & $1(4)$ \\
\hline CM = CaverMap ${ }^{\circledR}$. & & &
\end{tabular}

Table 4.

Risk Factors Analyzed for Interfering With Bilateral Hypogastric Nerve Identification During Dissection and CaverMap ${ }^{\circledR}$ Response

\begin{tabular}{|c|c|c|c|c|c|c|c|}
\hline \multirow[b]{2}{*}{ Factor } & \multirow[b]{2}{*}{ Total } & \multicolumn{3}{|c|}{ Surgeon Identification } & \multicolumn{3}{|c|}{ CM Response } \\
\hline & & Yes n (\%) & No $n(\%)$ & $P$ Value & Positive n (\%) & Negative n (\%) & $P$ Value \\
\hline \multicolumn{8}{|l|}{ Diagnosis } \\
\hline Rectal cancer & 13 & $8(61)$ & $5(39)$ & 0.99 & $11(85)$ & $2(15)$ & 0.99 \\
\hline Benign disease & 13 & $9(70)$ & $4(30)$ & & $10(77)$ & $3(23)$ & \\
\hline \multicolumn{8}{|l|}{ Radiation therapy } \\
\hline Yes & 9 & $6(67)$ & $3(33)$ & 0.99 & $8(89)$ & $1(11)$ & 0.58 \\
\hline $\begin{array}{l}\text { No } \\
\text { Procedure }\end{array}$ & \multicolumn{6}{|c|}{ Procedure } & \\
\hline LAR & 17 & $13(76)$ & $4(24)$ & 0.58 & $14(82)$ & $3(18)$ & 0.51 \\
\hline $\begin{array}{l}\text { APR } \\
\text { BMI }\end{array}$ & 9 & \multicolumn{5}{|c|}{ BMI } & \\
\hline$<30 \mathrm{~kg} / \mathrm{m}^{2}$ & 20 & $14(70)$ & $6(30)$ & 0.54 & $16(80)$ & $4(20)$ & 0.99 \\
\hline $30 \mathrm{~kg} / \mathrm{m}^{2}$ & 6 & $3(50)$ & $3(50)$ & & $5(83)$ & $1(17)$ & \\
\hline \multicolumn{8}{|l|}{ Previous surgery } \\
\hline Yes & 10 & $3(30)$ & $7(70)$ & 0.005 & $7(70)$ & $3(30)$ & 0.28 \\
\hline No & 16 & $14(88)$ & $2(13)$ & & $14(88)$ & $2(13)$ & \\
\hline
\end{tabular}

patients. In one patient, $\mathrm{CM}$ did not elicit a response from an identified nerve. After rectal excision, however, the CM response was positive in this patient. In the patient with unilateral response, CM successfully located the contralateral nerve.

In five (38.4 percent) patients, the cavernous nerves could not be identified without CM assistance. Among these patients, CM facilitated the identification in four (80 percent; 3 bilaterally and 1 unilaterally). CM failed to identify the cavernous nerves in only one patient, in whom identification of the hypogastric nerves was also not possible. The mean number of attempts to elicit an adequate response was 1.5 (range, 1-4).

\section{Nerve Stimulation After Proctectomy}

Table 3 summarizes the results of hypogastric and cavernous nerve stimulation at the completion of pelvic dissection. After removal of the specimen, the surgeon was able to identify the hypogastric nerves in all 29 patients. CM successfully confirmed the preservation of these nerves in 28 (96.6 percent) patients, at least in one side. CM failed to confirm preservation of both hypogastric nerves in only one patient, the same patient in whom the surgeon could not visualize nor $\mathrm{CM}$ confirm its integrity during dissection. The cavernous nerves were identified by the surgeon in 26 of 27 cases in at least one side, at the completion of dissection. CM confirmed parasympathetic function in all nerves (23 bilateral; 3 unilateral) except in one case; this patient did not participate in the protocol during dissection.

\section{Factors Affecting Nerve Identification During TME Dissection}

A summary of the factors possibly interfering with the surgeons ability to identify the hypogastric and cavernous nerves during dissection is shown in Tables 4 and 5, respectively. CM results are displayed as well. The analysis demonstrates that the hypogastric nerves were more likely to be identified bilaterally if the patient did not have a previous surgery, including mobilization of the rectum or dissection 
Table 5.

Risk Factors Analyzed for Interfering With Bilateral Cavernous Nerve Identification During Dissection and CaverMap $^{\circledR}$ Response

\begin{tabular}{|c|c|c|c|c|c|c|c|}
\hline \multirow[b]{2}{*}{ Factor } & \multirow[b]{2}{*}{ Total } & \multicolumn{3}{|c|}{ Surgeon Identification } & \multicolumn{3}{|c|}{$\mathrm{CM}$ response } \\
\hline & & Yes n (\%) & No $n(\%)$ & $P$ Value & Positive n (\%) & Negative n (\%) & $P$ Value \\
\hline \multicolumn{8}{|l|}{ Diagnosis } \\
\hline Rectal cancer & 7 & $5(71)$ & $2(29)$ & 0.27 & $4(50)$ & $3(60)$ & 0.99 \\
\hline Benign disease & 6 & 2 (33) & $4(67)$ & & $4(67)$ & $2(33)$ & \\
\hline \multicolumn{8}{|l|}{ Radiation therapy } \\
\hline Yes & 6 & $4(67)$ & $2(33)$ & 0.58 & $3(50)$ & $3(50)$ & 0.51 \\
\hline No & 7 & $3(43)$ & $4(57)$ & & $5(71)$ & $2(29)$ & \\
\hline \multicolumn{8}{|l|}{ Procedure } \\
\hline LAR & 6 & $4(67)$ & $2(33)$ & 0.58 & $3(50)$ & $3(50)$ & 0.51 \\
\hline \multicolumn{8}{|l|}{ BMI } \\
\hline$<30 \mathrm{~kg} / \mathrm{m}^{2}$ & 9 & $5(44)$ & $4(56)$ & 0.46 & $5(56)$ & $4(44)$ & 0.99 \\
\hline $30 \mathrm{~kg} / \mathrm{m}^{2}$ & 4 & $3(75)$ & $1(25)$ & & $3(75)$ & $1(25)$ & \\
\hline \multicolumn{8}{|l|}{ Previous surgery } \\
\hline Yes & 6 & $2(33)$ & $4(67)$ & 0.27 & $4(67)$ & $2(33)$ & 0.99 \\
\hline No & 7 & $5(71)$ & $2(29)$ & & $4(57)$ & $3(43)$ & \\
\hline
\end{tabular}

close to it. Of the 10 patients who had undergone previous surgery only 3 (30 percent; 95 percent confidence interval, 7 percent, 65 percent) had the nerves bilaterally identified, in contrast to 14 of 16 (82.3 percent) patients who did not have a history of prior surgery $(P=0.005)$. Of the ten patients who had undergone previous surgery, CM confirmed or enhanced nerve identification in seven (70 percent). Table 6 lists the previous procedures and surgical indications. None of the other factors analyzed affected identification or confirmation of the autonomic nerves; no adverse events were observed with this device.

\section{DISCUSSION}

Preservation of the pelvic autonomic nerves during rectal surgery, to avoid postoperative sexual dysfunction, is a well-recognized challenge. Several authors have focused on this problem and in a review of 19 studies $^{8}$ it appeared that approximately one-quarter of male patients suffered from erectile dysfunction after conventional surgery for rectal cancer and loss of ejaculation was found in 16 percent of the cases. In a recent prospective study specifically focusing on pelvic nerve preservation during TME, the results were slightly, but not dramatically, improved..$^{9}$ Thus, even in the hands of experienced surgeons, the exact course of a pelvic nerve may be difficult to assess, particularly in patients with advanced malignant disease or previous surgery. The current study fo- cused on use of a device with the potential ability to enhance pelvic autonomic nerve identification and preservation in the male patient during rectal surgery.

$\mathrm{CM}$ combines a nerve stimulator with a penile sensor that detects slight changes of 0.5 percent in penile girth on stimulation, indicating nerve integrity. Kim et $a l .{ }^{10}$ found that a response of only 0.5 percent correlated with a number of false-positive results. When a 1 percent cutoff value was used, however, no falsepositive response was obtained. Although a minimal change of 0.5 percent was considered a positive response in the current study, over 90 percent $(50 / 55)$ of stimulated sympathetic bundles and 95.5 percent (47/49) of parasympathetic bundles with a positive response had a tumescence of 1 percent or greater at the end of surgery. In addition, we have arbitrarily used CM to stimulate structures other than neural structures, with no response being elicited. Finally, it is interesting to note there was a satisfactory correlation between preoperative erectile function and a 0.5 percent CM response, as 95 percent of patients who were able to have intercourse before surgery had bilateral cavernous nerves response elicited by $\mathrm{CM}$, whereas the majority ( 75 percent) of patients not able to have intercourse had only unilateral or no CM response.

The present results confirm the well-known fact that the hypogastric nerves, located at the pelvic sidewalls, are far easier to detect than the cavernous nerves. Nevertheless, in approximately one-third of 
Table 6.

Previous Surgery and Surgery Indication

\begin{tabular}{|c|c|c|}
\hline Procedure & $\begin{array}{c}\text { No. of } \\
\text { Patients }\end{array}$ & Surgery Indication \\
\hline Total colectomy + ileorectal anastomosis & 3 & Familial adenomatous polyposis and ulcerative colitis \\
\hline Total colectomy + ileostomy & 1 & Fulminant colitis \\
\hline Rectosigmoidectomy & 1 & Rectal cancer \\
\hline Proctectomy & 1 & Rectal cancer \\
\hline Pouch excision & 1 & Pouch failure \\
\hline Prostatectomy & & Prostate cancer \\
\hline
\end{tabular}

the patients, the surgeon was not able to bilaterally identify the hypogastric nerves during dissection. With regard to the cavernous nerves located at the posterolateral aspect of the seminal vesicles, that the surgeon elected not to interfere with the dissection to locate these nerves in 16 cases may reflect the tedious task of identifying these nerves at a critical and demanding point of the rectal dissection. This feature may be of particular importance, because injury to the cavernous nerves at this level may impair both erection and ejaculation function. In this study, pelvic nerve identification was possible in 60 percent of patients in whom it was attempted. Thus, we demonstrated that pelvic nerve identification during rectal surgery could potentially be improved.

CM emerges in this study as a potentially reliable tool to enhance and confirm nerve localization. The device confirmed the function of all hypogastric identified nerves, and furthermore facilitated the nerve identification in another 11 cases. CM failed to improve identification of only one of the cases with unidentified nerves. Moreover, the current results show that $\mathrm{CM}$ was able to identify the cavernous nerves in five of six patients in whom it was unidentified during surgery. Regardless of nerve position, CM was able to identify the contralateral nerve in all cases in which the surgeon made a unilateral identification during dissection. Finally, CM was a useful tool in objectively confirming the preservation of the autonomic nerves in 27 (93 percent) patients, at least unilaterally, at the end of the procedure.

Of the several factors analyzed that might have a possible impact on the surgeons ability to identify autonomic nerves and on the ability of CM to confirm nerve function, only previous surgery was demonstrated to have an impact on the identification rate of the hypogastric nerves during dissection $(P=0.005)$. Thus, among the nine patients in whom the hypogastric nerves were not bilaterally identified, seven (78 percent) had a history of previous surgery. The fact that no other factor was found to have a significant impact may partially be explained by the relatively small number of patients in this series. Nevertheless, our findings corroborate those of Junginger et al., ${ }^{9}$ who, in their evaluation of pertinent factors for incomplete identification of the pelvic autonomic nerves, found that previous dissection and intraoperative blood loss $>1,000 / \mathrm{ml}$ exerted an independent influence on pelvic nerve identification.

Only one previous study has evaluated the potential benefits of CM during TME. ${ }^{11}$ This study, however, was not designed to assess CM as a nerve-identifying tool, but focused on its ability to predict sexual function after surgery. The study included 21 patients undergoing low anterior resection for rectal cancer and only the parasympathetic nerves were stimulated; the surgeon was able to identify the nerve structures in all patients. No false-positive tumescence responses were observed, resulting in 100 percent specificity. Of the 20 patients considered to have intact parasympathetic nerves after TME, 17 had bilateral tumescence response to nerve stimulation whereas the other 3 had unilateral response. At six-month follow-up, 94 percent of the patients with at least unilateral response displayed normal sexual function. Because the aim of our study was to asses the efficacy of CM in the identification of the autonomic nerves, the ability of CM to preserve the nerves or its impact on improved functional outcome was beyond the scope of the current study. However, a short analysis of our patients available at 12 months of follow-up (nonpublished results) demonstrated that of 21 patients with bilateral intact cavernous nerves, 14 were fully potent (able to have intercourse), 7 had some degree of impotence, and none was completely impotent. In contrast, none of the four patients who lacked bilateral response were fully potent. Calculations on the ability of a bilateral $\mathrm{CM}$ response to predict full potency resulted in a sensitivity of 100 percent, specificity of 36 percent, positive predictive value of 66 percent, and negative pre- 
dictive value of 100 percent. The low specificity rate reflects the fact that despite bilateral nerve integrity, a number of patients still have an inability to maintain intercourse after surgery. This may suggest that sexual function after surgery is a composite issue that includes not only the anatomic preservation of autonomic nerves within the pelvis but also a variety of other aspects.

Our results demonstrate that CM may potentially increase the identification rate of autonomic nerves during TME. Stimulating both the hypogastric and cavernous nerves added an average of 10 minutes to the surgery; however, this was not specifically evaluated in this study. Although CM is approved by the U.S. Food and Drug Administration for use during rectal surgery, the ultimate role of this device requires further evaluation. CM can be used in cases of advanced disease, previous surgery, or as an intraoperative tool during surgical training for facilitation of nerve identification. The cavernous nerves are more difficult to identify than the hypogastric nerves, therefore, it is probably at this site that CM may have the most substantial impact. Furthermore, in a patient with postoperative sexual dysfunction, a previously adequate CM response may be useful as "proof" of nerve preservation. In this situation, surgeons and patients can concentrate on seeking explanations for nerve damage other than impaired function.

\section{CONCLUSION}

Cavermap ${ }^{\circledR}$ may be a useful tool to facilitate identification of the pelvic autonomic nerves during TME and to objectively confirm nerve preservation.

\section{REFERENCES}

1. Havenga K, Maas CP, DeRuiter MC, Welvaart K, Trimbos JB. Avoiding long-term disturbance to bladder and sexual function in pelvic surgery, particularly with rectal cancer. Semin Surg Oncol 2000;18:235-43.

2. Tiainen J, Matikainen M, Hiltunen KM. Ileal J-pouchanal anastomosis, sexual dysfunction, and fertility. Scand J Gastroenterol 1999;34:185-8.

3. Maas CP, Moriya Y, Steup WH, Kiebert GM, Kranenbarg WM, Velde CJ. Radical and nerve-preserving surgery for rectal cancer in the Netherlands: a prospective study on morbidity and functional outcome. Br J Surg 1998;85: 92-7.

4. Heald RJ, Husband EM, Ryall RD. The mesorectum in rectal cancer surgery-the clue to pelvic recurrence? Br J Surg 1982;69:613-6.

5. Havenga K, Enker WE. Autonomic nerve preserving total mesorectal excision. Surg Clin North Am 2002;82: 1009-18.

6. Klotz L, Heaton J, Jewett M, et al. A randomized phase 3 study of intraoperative cavernous nerve stimulation with penile tumescence monitoring to improve nerve sparing during radical prostatectomy. J Urol 2000;164: 1573-8.

7. Orkin BA. Rectal carcinoma: treatment. In: Beck DE, Wexner SD, eds. Fundamentals of anorectal surgery. New York: McGraw-Hill, 1992:260-370.

8. Kim NK, Aahn TW, Park JK, et al. Assessment of sexual and voiding function after total mesorectal excision with pelvic autonomic nerve preservation in males with rectal cancer. Dis Colon Rectum 2002;45:1178-85.

9. Junginger T, Kneist W, Heintz A. Influence of identification and preservation of pelvic autonomic nerves in rectal cancer surgery on bladder dysfunction after total mesorectal excision. Dis Colon Rectum 2003;46:621-8.

10. Kim HL, Stoffel DS, Mhoon DA, Brendler CB. A positive CaverMap response poorly predicts recovery of potency after radical prostatectomy. Urology 2000;56: 561-4.

11. Hanna NN, Guillem J, Dosoretz A, Steckelman E, Minsky BD, Cohen AM. Intraoperative parasympathetic nerve stimulation with tumescence monitoring during total mesorectal excision for rectal cancer. J Am Coll Surg 2002;195:506-12. 